\section{Epidemiologie und Kosten des Bronchialkarzinoms in Deutschland}

Zusammenfassung: Zielsetzung: Das Bronchialkarzinom besitzt in den Industrieländern unter den malignen Erkrankungen bei Männern die höchste und bei Frauen eine zunehmende Inzidenz. Wir führten eine Krankheitskostenstudie für Deutschland durch, um die volkswirtschaftliche Bedeutung dieser Erkrankung zu untersuchen und die wichtigsten Kostenfaktoren zu identifizieren. Methoden: Das Referenzjahr für die Kostenanalyse bildete das Jahr 1996. In einer retrospektiven Analyse ermittelten wir direkte und indirekte Kosten mit Hilfe von Sekundärdaten öffentlicher Einrichtungen und der Pharmazeutischen Industrie. Die direkten Kosten wurden aus der Kostenperspektive der Krankenversicherung bestimmt. Zur Berechnung der indirekten Kosten griffen wir auf den Humankapitalansatz zurück. Resultate: Die jährlichen Gesamtkosten des Bronchialkarzinoms betrugen 8,31 Mrd. DM. Die indirekten Kosten erreichten mit 7,40 Mrd. DM einen überragenden Anteil von $89 \%$ an den Gesamtkosten. Der größte Kostenfaktor der indirekten Kosten, der vorzeitige Tod, erzielte alleine mit 4,85 Mrd. DM einen Anteil von $58 \%$ an den Gesamtkosten. Wichtigster Kostenfaktor der direkten Kosten war die stationäre Akutbehandlung, welche mit 0,85 Mrd. DM 93\% der direkten Kosten verursachte. Schlussfolgerung: Der hohe Anteil der indirekten Kosten an den Gesamtkosten, insbesondere geschuldet dem vorzeitigen Tod, ist das auffälligste Ergebnis unserer Krankheitskostenstudie des Bronchialkarzinoms. Vor diesem Hintergrund und angesichts der ätiologischen Bedeutung des inhalativen Nikotinkonsums für das Bronchialkarzinom plädieren wir dafür, die indirekten Kosten in Analysen zu den volkswirtschaftlichen Kosten des Rauchens einzubeziehen.

Epidemiology and Costs of Lung Cancer in Germany: Objective: Lung cancer shows the leading incidence of all cancers among men in the developed world and an increasing incidence among women. We performed a cost of illness study that aimed to assess the economic burden of lung cancer in Germany and to identify the main cost drivers. Methods: Costs were estimated for the year 1996. In a retrospective analysis we calculated direct and indirect costs based on secondary data from governmental institutions as well as from the pharmaceutical industry. We chose the cost perspective of sickness funds to estimate direct costs. The human capital approach was applied for the calculation of indirect costs. Results: Total estimated costs were DM 8.31 billion per year. The indirect costs of DM 7.40 bil-

Pneumologie 2001; 55: 333-338

(c) Georg Thieme Verlag Stuttgart · New York ISSN 0934-8387

\author{
D. Weißflog ${ }^{1}$, H. Matthys ${ }^{1}$, J. Hasse ${ }^{2}$, J. Chr. Virchow jr. ${ }^{1}$ \\ ${ }^{1}$ Abteilung für Pneumologie, Medizinische Klinik, \\ Albert-Ludwigs-Universität Freiburg \\ (Ärztlicher Direktor Prof. Dr. med. H. Matthys) \\ ${ }^{2}$ Abteilung für Lungenchirurgie, Chirurgische Klinik, \\ Albert-Ludwigs-Universität Freiburg \\ (Ärztlicher Direktor Prof. Dr. med. J. Hasse)
}

lion accounted for $89 \%$ of total estimated costs. The most important cost driver of the indirect costs, early death, represented on its own DM 4.85 billion, according to $58 \%$ of total estimated costs. Of the direct costs, $93 \%$ were due to hospitalization, amounting to DM 0.85 billion. Conclusions: This cost of illness study concerning lung cancer illustrates the outstanding importance of the indirect costs, mostly due to early death, for total costs. Based on these findings and on the leading role of smoking in the etiology of lung cancer, we suggest that studies dealing with the net costs of smoking to society should include indirect costs.

\section{Einleitung}

Das Bronchialkarzinom bildet in den Industrieländern die häufigste maligne Erkrankung bei Männern. Für Deutschland wird geschätzt, dass im Jahre 199728200 Männer neu an einem Bronchialkarzinom erkrankten. Die Inzidenz des Bronchialkarzinoms bei Frauen betrug im gleichen Jahr 8900 . Somit besaß in Deutschland das Bronchialkarzinom unter den malignen Erkrankungen bei den Frauen die vierthöchste Inzidenz nach Mammakarzinom, Kolon- und Uteruskarzinom. Allerdings vollzog sich bei Frauen in den letzten Jahren eine deutliche Zunahme der Inzidenz des Bronchialkarzinoms [1]. In einer weltweiten Schätzung wird angenommen, dass das Bronchialkarzinom aktuell an 10. Stelle der häufigsten Todesursachen steht und bis zum Jahr 2020 auf die 5. Position vorrücken wird [2]. Den wichtigsten ätiologischen Faktor des Bronchialkarzinoms stellt der inhalative Nikotinkonsum dar, welcher für 85-90\% aller Fälle von Bronchialkarzinomen verantwortlich gemacht wird [3]. Die Prognose des Bronchialkarzinoms richtet sich insbesondere nach seinem Stadium zum Zeitpunkt der Diagnosestellung. In einem Kollektiv von 187 operativ behandelten Patienten mit einem Bronchialkarzinom [4] variierte die Überlebenswahrscheinlichkeit stadienabhängig nach 1 Jahr von $52,8 \%$ bis $83,7 \%$ (durchschnittlich $74,3 \%$ ) und nach 5 Jahren von $18,9 \%$ bis $53,8 \%$ (durchschnittlich 39,5\%). Bei zum Diagnosezeitpunkt inoperablen Patienten bewegte sich die 5-Jahres-Überlebenswahrscheinlichkeit zwischen $8 \%$ und weniger als $1 \%$ [5]. Durchschnittliche Überlebensraten liegen aus einem Krebsregister des Saarlandes aus den 80er Jahren vor. Sie zeigen auf, dass bei Männern 1 Jahr bzw. 5 Jahre nach der Diagnosestellung des Bronchialkarzinoms 29\% bzw. 9\% der Erkrankten überlebten. Die relativen Überlebensraten bei den Frauen betrugen 34\% bzw. $17 \%$ [1]. Bemerkenswerterweise scheint sich die 5-Jahres- 
Überlebenswahrscheinlichkeit in den zurückliegenden vier Dekaden in Europa nicht wesentlich geändert zu haben [6].

Obwohl das Bronchialkarzinom eine große epidemiologische Bedeutung besitzt, gibt es weltweit nur wenige Studien, welche die volkswirtschaftliche Dimension dieser Erkrankung thematisieren. Vergleichsweise mehr Studien evaluieren die Kosteneffektivität unterschiedlicher diagnostischer und therapeutischer Algorithmen wie z.B. Alternativen zum mediastinalen Staging von Patienten mit einem nicht-kleinzelligen Bronchialkarzinom [7], Alternativen der Chemotherapie [810] oder unterschiedliche Follow-up-Regime [11]. Nach unserem Kenntnisstand sind nur wenige Studien zu den Gesamtkosten, die das Bronchialkarzinom in einem Staat pro Jahr verursacht, veröffentlicht worden. Es liegen Publikationen für Kanada [12], die USA [13] und die Niederlande [14] vor. Darüber hinaus existieren Angaben zu den durchschnittlichen Kosten pro Patienten mit einem Bronchialkarzinom pro Jahr oder vom Zeitpunkt der Diagnosestellung bis zum Tod aus Australien [15], Frankreich [16], Großbritannien [17], Kanada [12], den Niederlanden [18] und den USA [19]. Für Deutschland wurde im Weißbuch Lunge eine Schätzung der jährlichen Gesamtkosten des Bronchialkarzinoms für das Jahr 1992 in Höhe von 5,4 Mrd. DM [20] und in einer aktualisierten Ausgabe [21] für das Jahr 1997 in Höhe von 5,32 Mrd. DM angegeben. Auf die indirekten Kosten entfiel der größte Kostenanteil. Wichtigster Kostenfaktor war der vorzeitige Tod. Ruff u. Mitarb. untersuchten die ökonomische Bedeutung des Rauchens in Deutschland [22]. Im Rahmen dieser Analyse ermittelten sie für das Jahr 1996 durch das Rauchen verursachte Gesamtkosten des Bronchialkarzinoms von 5,08 Mrd. DM (2,59 Euro) bzw. ohne Berücksichtigung des attributiven Risikos des Rauchens 5,71 Mrd. DM. Die indirekten Kosten stellten auch in dieser Studie den größten Kostenanteil. Einzelne Kostenfaktoren der direkten und der indirekten Kosten wurden nicht separat für das Bronchialkarzinom aufgeführt. Wir intendierten eine detaillierte Krankheitskostenstudie des Bronchialkarzinoms für Deutschland mit transparenten methodischen Annahmen.

\section{Methodik}

Bei unserer Krankheitskostenstudie handelt es sich um eine retrospektive Analyse. Sie stützt sich auf Sekundärdaten. Es wurden direkte und indirekte Kosten eingeschlossen. Direkte Kosten entstehen aus dem Verbrauch an Gütern und Dienstleistungen durch diagnostische und therapeutische Maßnahmen. In Deutschland werden die direkten Kosten in erster Linie von den Krankenkassen getragen. Wir wählten daher die Kostenperspektive der Krankenkassen und untersuchten die direkten Kosten, die den Krankenkassen infolge des Bronchialkarzinoms entstanden. Diese Vorgehensweise impliziert, dass wir diejenigen direkten Kosten, welche nicht von den Krankenkassen getragen werden, auch nicht erfassen, wie z.B. die Selbstmedikation von Patienten. Aus volkswirtschaftlicher Sicht entstehen durch Erkrankungen nebst den direkten Kosten aus dem Verlust an menschlicher Produktivität durch Morbidität und Mortalität weitere Kosten. Für die Berechnung dieser indirekten Kosten wendeten wir den Humankapitalansatz an. Dieser nimmt eine monetäre Bewertung des Produktivitätsverlustes der erwerbstätigen Bevölkerung mit dem durchschnittlichen Bruttoeinkommen vor. Trotz unterschiedlicher Kritikpunkte am Humankapitalansatz werden die in- direkten Kosten in den meisten Studien nach dieser Methode ermittelt [23]. Als Referenzjahr für die Kostenanalyse setzten wir das Jahr 1996 fest, da dieses Jahr das aktuellste Kalenderjahr war, für das Daten in allen von uns erfassten Bereichen vorlagen. Die verwendeten Sekundärdaten waren nach ICD (International Classification of Diseases) verschlüsselt, folglich orientierten wir unsere Analyse ebenfalls an dieser Klassifikation in der 9. Version. Das Bronchialkarzinom wurde unter der ICD-Nummer 162 erfasst.

Unter den direkten Kosten berücksichtigten wir in unserer Studie die Kosten der stationären Akutbehandlung, die Kosten der stationären Rehabilitation und die der ambulanten Arzneimittelverordnungen. Unberücksichtigt blieben die Kosten der ambulanten ärztlichen Leistungen, da hierfür nach unserem Kenntnisstand keine Daten vorlagen, die eindeutig der ICD-Nummer 162 zugeordnet werden konnten. Zur Bestimmung der Kosten der stationären Akutbehandlung entnahmen wir die Fallzahl je 10000 Versicherte und die Anzahl der Tage je Fall der AOK-Krankheitsartenstatistik 1996 [24]. Unter Verwendung des Statistischen Jahrbuches [25] extrapolierten wir diese Daten auf die Gesamtbevölkerung der Bundesrepublik Deutschland hoch. Wir trafen hierbei die Annahme, dass die Daten der AOK-Krankheitsartenstatistik repräsentativ für alle Krankenversicherten in Deutschland sind, unter Einschluss der privat Krankenversicherten, welche ohnehin nur einen Anteil von ca. 10\% an der Gesamtheit der Krankenversicherten stellen [1]. Innerhalb der Gesetzlichen Krankenversicherung weisen die Allgemeinen Ortskrankenkassen mit $40 \%$ den höchsten Versichertenanteil auf. Da in der Bundesrepublik Deutschland der überwiegende Teil der Bevölkerung krankenversichert ist, kann man ferner grosso modo die Gesamtheit der krankenversicherten Personen mit der Bevölkerung gleichsetzen. Im Jahre 1996 wurde die stationäre Akutbehandlung von Patienten mit den von uns betrachteten Diagnosen über Pflegetage abgerechnet. Die durchschnittlichen Kosten je Pflegetag entnahmen wir einer Veröffentlichung des Bundesministeriums für Gesundheit [1]. Zur Bestimmung der Kosten der stationären Rehabilitation erhielten wir die Fallzahlen an stationären Rehabilitationen von einer Datenbank des Verbandes Deutscher Rentenversicherungsträger [26]. Die Kosten pro stationärer Rehabilitation variierten je nach Vertrag der einzelnen Rehabilitationsklinik mit dem jeweiligen Kostenträger. Wir nahmen aus den uns vorliegenden Angaben eine Schätzung der durchschnittlichen Kosten pro stationärem Rehabilitationsaufenthalt vor. Die Anzahl der ambulanten Verordnungen und deren Kosten entnahmen wir Statistiken der Pharmazeutischen Industrie [27]. Wir verwendeten hierbei Kostendaten, die auf dem Apothekenverkaufspreis basierten.

Unter den indirekten Kosten erfassten wir solche, die aus (vorübergehender) Arbeitsunfähigkeit, Frühberentung und vorzeitigem Tod (d.h.: Tod vor dem regulären Berentungsalter) entstanden. Für die Ermittlung der indirekten Kosten verwendeten wir den Humankapitalansatz. Zur Berechnung der Kosten der Arbeitsunfähigkeit entnahmen wir die Zahl der Arbeitsunfähigkeitsfälle je 10000 Versicherte der AOK-Krankheitsartenstatistik 1996. Unter Verwendung des Statistischen Jahrbuches extrapolierten wir diese Daten auf die Gesamtbevölkerung der Bundesrepublik Deutschland hoch. Wir trafen hierbei die Annahme, dass die Daten der AOK-Krankheitsartenstatistik repräsentativ für alle Krankenversicherten bzw. 
grosso modo für die Gesamtbevölkerung in Deutschland sind. Die Kosten pro Tag Arbeitsunfähigkeit kalkulierten wir mit dem durchschnittlichen jährlichen Bruttoeinkommen je beschäftigtem Arbeitnehmer, das wir dem Statistischen Jahrbuch entnahmen, dividiert durch die Anzahl der Jahrestage. Zur Berechnung der Kosten der Frühberentung entnahmen wir die Anzahl der Rentenzugänge, gegliedert nach Altersklassen, der VDR-Statistik Rentenzugang [28]. Für Frauen und Männer getrennt errechneten wir die Anzahl der verlorenen Jahre Erwerbstätigkeit. Wir nahmen hierbei an, dass Frauen mit 20 Jahren in das Erwerbsleben eintreten und mit 62 Jahren in Rente gehen, Männer mit 20 Jahren in das Erwerbsleben eintreten und mit 65 Jahren in Rente gehen. Die Anzahl der verlorenen Jahre Erwerbstätigkeit multiplizierten wir mit dem durchschnittlichen jährlichen Bruttoeinkommen je beschäftigtem Arbeitnehmer [25], um die Kosten der Frühberentung zu erhalten. Zur Berechnung der Kosten des vorzeitigen Todes entnahmen wir die Anzahl der Todesfälle im erwerbsfähigen Alter, gegliedert nach Altersklassen, der Todesursachenstatistik [29]. Für Frauen und Männer getrennt errechneten wir die Anzahl der verlorenen Jahre Erwerbstätigkeit. Wir nahmen hierbei analog zur Berechnung der Kosten der Frühberentung an, dass Frauen mit 20 Jahren in das Erwerbsleben eintreten und mit 62 Jahren in Rente gehen, Männer mit 20 Jahren in das Erwerbsleben eintreten und mit 65 Jahren in Rente gehen. Die Anzahl der verlorenen Jahre Erwerbstätigkeit aufgrund vorzeitigen Todes korrigierten wir um die durchschnittliche Erwerbsquote in den jeweiligen Altersklassen, welche wir dem Statistischen Jahrbuch entnahmen [25]. Diese Größe multiplizierten wir mit dem durchschnittlichen jährlichen Bruttoeinkommen je beschäftigtem Arbeitnehmer, um die Kosten des vorzeitigen Todes zu erhalten.

\section{Resultate}

Direkte Kosten

In Deutschland waren 1996132522 Fälle mit einem Bronchialkarzinom zu verzeichnen, welche stationär in einem Akutkrankenhaus behandelt wurden. Dies entsprach unter den oben erläuterten Annahmen 16,2 Fällen je 10000 Versicherte. Die durchschnittliche stationäre Aufenthaltsdauer betrug 11,8 Tage, so dass sich 1,56 Mio. Pflegetage ergaben. Bei durchschnittlichen Krankenhauskosten 1996 je Pflegetag von 542,60 DM resultierten hieraus Kosten der stationären Akutbehandlung von Patienten mit einem Bronchialkarzinom in Höhe von 847 Mio. DM (vgl. Abb.1). Im Bereich der stationären Rehabilitation waren im Jahre 19965221 Fälle mit einem Bronchialkarzinom erfasst. Es ergaben sich hieraus bei durchschnittlichen Kosten pro Rehabilitationsaufenthalt von ca. 7000 DM Kosten der stationären Rehabilitationsbehandlung von 37 Mio. DM (vgl. Abb.1). Die Anzahl der ambulanten Arzneimittelverordnungen belief sich im Jahre 1996 auf 0,34 Mio. für die Diagnose Bronchialkarzinom. Hieraus entstanden Kosten in Höhe von 27 Mio. DM (vgl. Abb.1). Dies entsprach durchschnittlichen Kosten pro Verordnung von 78,26 DM. Insgesamt beliefen sich die direkten Kosten des Bronchialkarzinoms in Deutschland 1996 auf 0,91 Mrd. DM. Der wichtigste Kostenfaktor war mit deutlichem Abstand die stationäre Akutbehandlung. Diese erreichte einen Anteil von 93\% an den direkten Kosten. Demgegenüber waren die Kostenanteile der stationären Rehabilitation und der ambulanten Arzneimittelverordnungen nahezu unbedeutend. Die

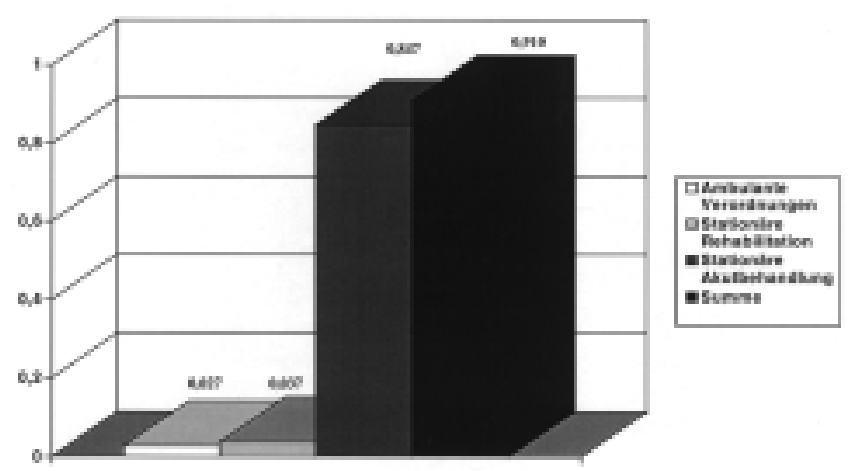

Abb. 1 Direkte Kosten (in Mrd. DM).

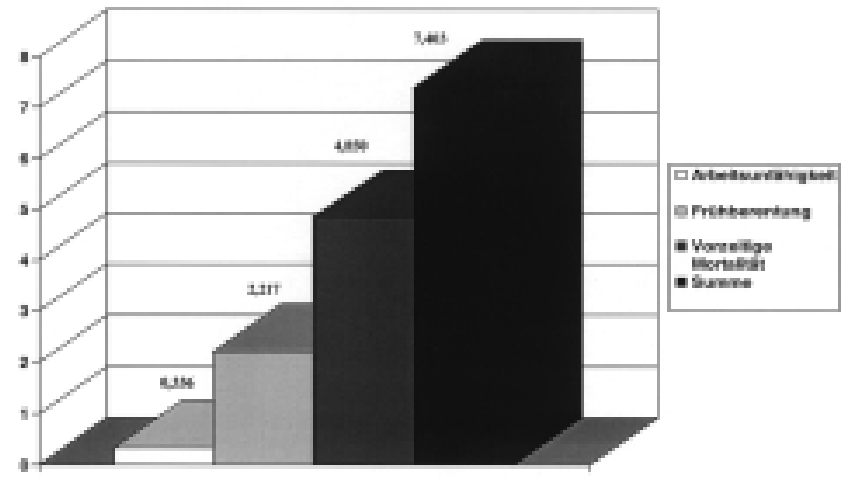

Abb. 2 Indirekte Kosten (in Mrd. DM).

stationäre Rehabilitation erzielte einen Anteil an den direkten Kosten von 4\% und die ambulanten Arzneimittelverordnungen einen Anteil von $3 \%$.

\section{Indirekte Kosten}

In Deutschland wurden für das Jahr 199626382 Fälle an Arbeitsunfähigkeit aufgrund eines Bronchialkarzinoms erfasst. Dies entsprach 3,2 Fällen je 10000 Versicherte. Die durchschnittliche Dauer je Fall von Arbeitsunfähigkeit betrug 75,1 Tage. Somit resultierten absolut 1980789 Tage an Arbeitsunfähigkeit. Bei Multiplikation dieser Zahl mit dem durchschnittlichen täglichen Bruttoeinkommen je beschäftigten Arbeitnehmer von 169,59 DM beliefen sich die Kosten der Arbeitsunfähigkeit verursacht durch Bronchialkarzinome auf 336 Mio. DM (vgl. Abb. 2). Über die Verteilung der Rentenzugänge, untergliedert nach Altersklassen und Geschlecht, in Deutschland im Jahre 1996 gibt Tab.1 Auskunft. In den aufgeführten Altersklassen wurden insgesamt 3431 Fälle von Frühberentung aufgrund von Bronchialkarzinomen erfasst. Hierbei handelte es sich in 562 Fällen um Frauen. Wir errechneten insgesamt 35816 verlorene Jahre Erwerbstätigkeit in Folge der Frühberentung unter der Annahme, dass Frauen mit 20 Jahren in das Erwerbsleben eintreten und mit 62 Jahren in Rente gehen, Männer mit 20 Jahren in das 
Tab. 1 Rentenzugänge wegen verminderter Erwerbsfähigkeit

\begin{tabular}{|c|c|c|c|c|c|c|c|}
\hline & $\begin{array}{l}\text { Alterskl } \\
\text { bis } 39\end{array}$ & $\begin{array}{l}\text { ahre) } \\
40-44\end{array}$ & $45-49$ & $50-54$ & $55-59$ & $\begin{array}{l}\text { ab } 60 \\
\text { (bis } 62 \text { bei Frauen) } \\
\text { (bis } 65 \text { bei Männern) }\end{array}$ & $\begin{array}{l}\text { Summe } \\
\text { (alle Altersstufen) }\end{array}$ \\
\hline Frauen & 30 & 43 & 99 & 167 & 202 & 21 & 562 \\
\hline Männer & 49 & 129 & 318 & 736 & 1318 & 319 & 2869 \\
\hline Insgesamt & 79 & 172 & 417 & 903 & 1520 & 340 & 3431 \\
\hline
\end{tabular}

Daten beziehen sich auf die Gesetzliche Rentenversicherung

Quelle: VDR-Statistik Rentenzugang 1996. Hrsg. Verband Deutscher Rentenversicherungsträger, Mai 1997

Tab. 2 Todesfälle im erwerbsfähigen Alter

\begin{tabular}{|c|c|c|c|c|c|c|c|c|c|c|}
\hline & \multicolumn{10}{|c|}{ Altersklassen (Jahre) } \\
\hline & $20-25$ & $25-30$ & $30-35$ & $35-40$ & $40-45$ & $45-50$ & $50-55$ & $55-60$ & $\begin{array}{l}\text { ab } 60 \\
\text { (bis } 62 \text { bei Frauen) } \\
\text { (bis } 65 \text { bei } \\
\text { Männern) }\end{array}$ & $\begin{array}{l}\text { Summe } \\
\text { (alle Altersstufen) }\end{array}$ \\
\hline Frauen & 0 & 3 & 27 & 69 & 177 & 286 & 464 & 762 & 431 & 2219 \\
\hline Männer & 5 & 12 & 32 & 100 & 318 & 797 & 1673 & 3597 & 4529 & 11063 \\
\hline Insgesamt & 5 & 15 & 59 & 169 & 495 & 1083 & 2137 & 4359 & 4960 & 13282 \\
\hline
\end{tabular}

Quelle: Todesursachen in Deutschland 1996. Hrsg. Statistisches Bundesamt. Gesundheitswesen. Fachserie 12, Reihe 4. Wiesbaden 1997

Erwerbsleben eintreten und mit 65 Jahren in Rente gehen. Bei einem durchschnittlichen jährlichen Bruttoeinkommen je beschäftigten Arbeitnehmer von 61900 DM im Jahre 1996 ergaben sich somit Kosten der Frühberentung aufgrund von Bronchialkarzinomen in Höhe von 2,22 Mrd. DM. Über die Verteilung der Todesfälle im erwerbsfähigen Alter, untergliedert nach Altersklassen und Geschlecht, in Deutschland im Jahre 1996 informiert Tab. 2. Es wurden insgesamt 13282 Todesfälle aufgrund von Bronchialkarzinomen im erwerbsfähigen Alter registriert, darunter 2219 Frauen. Wir errechneten unter der oben aufgeführten Annahme folglich insgesamt 103225 verlorene Jahre Erwerbstätigkeit, geschuldet dem vorzeitigen Tod. Da das Statistische Bundesamt nur Todesfälle für die Altersklasse 60-65 Lebensjahre ausweist, trafen wir bei Frauen die zusätzliche Annahme, dass für die Altersklasse 60-62 Lebensjahre die ausgewiesene Zahl approximativ halbiert werden kann. Bei einem durchschnittli-

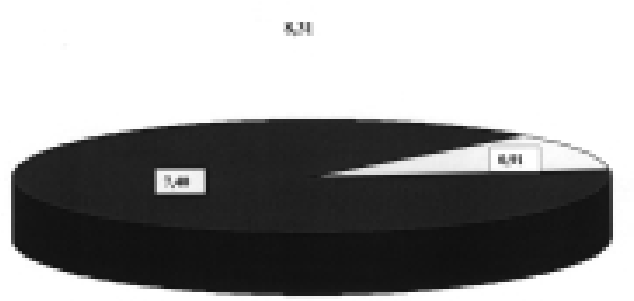

ODrebtis Kinsten

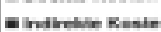

Abb. 3 Gesamtkosten (in Mrd. DM). chen jährlichen Bruttoeinkommen je beschäftigtem Arbeitnehmer von 61900 DM im Jahre 1996 und einer durchschnittlichen Erwerbsquote in den betrachteten Altersklassen von $76 \%$ ergaben sich auf diese Weise Kosten des vorzeitigen Todes durch Bronchialkarzinome in Höhe von 4,85 Mrd. DM (vgl. Abb. 2). In der Zusammenschau beliefen sich in Deutschland im Jahre 1996 die indirekten Kosten verursacht durch Bronchialkarzinome auf 7,40 Mrd. DM. Der vorzeitige Tod war mit einem Anteil von 65\% an den gesamten indirekten Kosten der mit Abstand wichtigste Kostenfaktor, gefolgt von Frühberentung (30\%) und Arbeitsunfähigkeit (5\%).

\section{Gesamtkosten}

Die Gesamtkosten des Bronchialkarzinoms ermittelten wir für Deutschland im Jahre 1996 mit 8,31 Mrd. DM (vgl. Abb. 3). Die indirekten Kosten erreichten mit 7,40 Mrd. DM den überragenden Anteil von $89 \%$ an den Gesamtkosten. Der größte Kostenfaktor der indirekten Kosten, der vorzeitig Tod, erzielte alleine einen Anteil von 58\% an den Gesamtkosten. Es bleibt anzumerken, dass wir ambulante ärztliche Leistungen als Faktor für die direkten Kosten mangels geeigneter Daten nicht erfassen konnten. Unsere Schätzung der direkten Kosten fällt daher eher zu niedrig aus.

\section{Diskussion}

Methodisch unterscheiden Goodwin u. Shepherd [7] zwei grundlegende ökonomische Forschungsansätze im Gesundheitswesen. Der „micro approach“ analysiert die ökonomische Vorteilhaftigkeit, etwa gemessen an der Kosteneffektivität, einzelner diagnostischer oder therapeutischer Vorgehensweisen wie z.B. unterschiedlicher Chemotherapieregime für 
bestimmte, definierte Patientenkollektive. Die meisten gesundheitsökonomischen Veröffentlichungen zum Thema Bronchialkarzinom lassen sich dieser Kategorie zuordnen. Die vorliegende Studie hingegen entspringt dem Kontext des „macro approach“. Sie ist als Krankheitskostenstudie zu klassifizieren und untersucht die Bedeutung der Erkrankung Bronchialkarzinom auf volkswirtschaftlicher Ebene für Deutschland. Die Daten beziehen sich auf das Jahr 1996. Wir ermittelten Gesamtkosten in Höhe von 8,31 Mrd. DM. Die direkten Kosten waren fast ausschließlich auf die stationäre Akutbehandlung mit 0,85 Mrd. DM zurückzuführen. Stationäre Rehabilitation und ambulante Arzneimittelverordnungen erlangten demgegenüber als Kostenfaktoren keine Bedeutung. Ambulante ärztliche Leistungen konnten wir mangels geeigneter Daten nicht erfassen. Als augenfälligstes Resultat imponierten jedoch die hohen indirekten Kosten in Höhe von 7,40 Mrd. DM. Dies entspricht einem Anteil der indirekten Kosten von $89 \%$ an den Gesamtkosten. Unter den indirekten Kosten war der wichtigste Kostenfaktor mit Abstand der vorzeitige Tod, auf welchen alleine $58 \%$ der Gesamtkosten fielen. Auf die Frühberentung gingen 26\% und auf Arbeitsunfähigkeit 5\% der Gesamtkosten zurück. In unserer Krankheitskostenstudie trafen wir die Annahme, dass die Daten der AOK-Krankheitsartenstatistik repräsentativ für alle Krankenversicherten in Deutschland, unter Einschluss der privat Krankenversicherten, sind. Wir können nicht ausschließen, dass durch diese Annahme, die wir aufgrund einer limitierten Datenlage getroffen haben, ein bias auf die von uns ermittelten Kosten ausgeht. Anzuführen wären in diesem Zusammenhang z.B. divergierende Inzidenzen des Bronchialkarzinoms unter den Mitgliedern einzelner Krankenversicherungen und abweichende Leistungs- und Kostenstrukturen für unterschiedliche Krankenversicherungen. Allerdings kann man bei der intransparenten Datenlage nur spekulieren, ob diese Einflussfaktoren zu höheren bzw. niedrigeren Kosten des Bronchialkarzinoms in Deutschland führen als die von uns ermittelten Kosten oder ob sie sich eventuell auch gegenseitig in ihrer Wirkung aufheben.

Das Weißbuch Lunge gab die geschätzten jährlichen Gesamtkosten des Bronchialkarzinoms in Deutschland für das Jahr 1992 mit 5,4 Mrd. DM [20] an, in einer aktualisierten Fassung [21] für 1997 mit dem unveränderten Betrag von 5,32 Mrd. DM. Der Umstand, dass die Kosten im Zeitablauf von 5 Jahren konstant geblieben sein sollen, scheint uns wenig plausibel zu sein. Die Differenz zu den von uns für das Jahr 1996 ermittelten Gesamtkosten von 8,31 Mrd. DM ist beachtlich. Sie beruht im Wesentlichen auf den indirekten Kosten, welche wir mit 7,40 Mrd. DM statt 4,19 Mrd. DM fast doppelt so hoch ermittelten. Dennoch überwogen auch gemäß Weißbuch Lunge - vergleichbar zu unseren Resultaten - die indirekten deutlich die direkten Kosten und die vorzeitigen Todesfälle bildeten den wichtigsten Kostenfaktor der indirekten Kosten. Soweit wir den allgemeinen methodischen Angaben des Weißbuch Lunge entnehmen, bestehen einige methodische Unterschiede zu unserer Studie, welche die Diskrepanz der geschätzten indirekten Kosten des Bronchialkarzinoms in Deutschland teilweise erklären könnten. Der durchschnittliche Verlust an Arbeitszeit infolge Frühberentung wurde im Weißbuch Lunge arbiträr mit 60 Monaten angenommen. Wir ermittelten die verlorenen Lebensjahre Erwerbstätigkeit aufgrund Frühberentung nicht arbiträr, sondern in einer detaillierten, altersklassenbezogenen Analyse. Hieraus resultierte ein etwa doppelt so hoher Verlust an Arbeitszeit durch Frühberentung im Vergleich zur Annahme im Weißbuch Lunge. Des Weiteren bildete die durchschnittliche Brutto-Lohn- und Gehaltssumme im Weißbuch Lunge die Grundlage zur Berechnung der indirekten Kosten. Wir verwendeten das (höher ausfallende) durchschnittliche jährliche Bruttoeinkommen je beschäftigtem Arbeitnehmer. Im Jahre 1996 betrug dieses 61900 DM. In einer aktuellen Publikation zu den Kosten des Rauchens in Deutschland setzten Ruff u. Mitarb. [22] für 1996 einen nahezu identischen Wert von 31633 Euro, entsprechend 61680 DM, an. Die Berechnung der Kosten der vorzeitigen Todesfälle erfolgte im Weißbuch Lunge unter Verwendung einer Abdiskontierungsrate um 4\%. Wir nahmen in unserer Studie implizit an, dass die Abdiskontierungsrate der Zuwachsrate der Bruttoeinkommen in den künftigen Jahren gleicht. Somit heben sich beide Effekte gegenseitig auf.

Ruff u. Mitarb. thematisierten die ökonomische Bedeutung des Rauchens in Deutschland [22]. Im Rahmen dieser Arbeit berechneten sie für das Jahr 1996 durch das Rauchen verursachte Gesamtkosten des Bronchialkarzinoms von 5,08 Mrd. DM (2,59 Mrd. Euro) bzw. ohne Berücksichtigung des attributiven Risikos des Rauchens 5,71 Mrd. DM. Obwohl Ruff u. Mitarb. analog zu unserer Vorgehensweise den Humankapitalansatz zur Berechnung der indirekten Kosten verwendeten und den identischen Betrag für das jährliche Bruttoeinkommen je Arbeitnehmer ansetzten, erhielten sie mit ca. 4,7 Mrd. DM ebenfalls deutlich niedrigere indirekte Kosten als wir mit 7,40 Mrd. DM. Inwieweit diese Diskrepanz auf Unterschieden im epidemiologischen Datengerüst oder auf anderen Einflussfaktoren beruht, kann mangels Angaben der zugrunde gelegten Fallzahlen nicht eruiert werden. Somit bleibt zu konstatieren, dass unsere Schätzung der jährlichen Gesamtkosten des Bronchialkarzinoms in Deutschland weitaus höher ausfällt als die bisher vorliegenden Angaben. Bei näherer Betrachtung lässt sich dieser Unterschied auf die indirekten Kosten eingrenzen, welche wir etwa doppelt so hoch ermittelten. Insofern kommt das bereits von anderen Autoren festgestellte Überwiegen der indirekten über die direkten Kosten in unserer Studie noch akzentuierter zum Ausdruck. Aus dem Ausland liegen Schätzungen zu den Gesamtkosten des Bronchialkarzinoms vor, die sich allerdings nur auf die direkten Kosten beziehen. Desch und Kollegen geben 1996 für die USA jährliche direkte Kosten von 7,8 Mrd. US \$ an [13]. Eine Untergliederung dieser Kosten wird nicht aufgeführt. Evans und Kollegen entwarfen für Kanada ein Mikrosimulationsmodell [12]. Sie berechneten für die 15624 Fälle von Bronchialkarzinomen, welche im Jahre 1988 in Kanada diagnostiziert wurden, direkte Kosten in den folgenden fünf Jahren in Höhe von 328 Mio. CDN \$. Hiervon entfielen bereits auf das erste Jahr 269 Mio. CDN \$. Die Hospitalisierungskosten bildeten den wichtigsten Kostenfaktor. Koopmanshap und Kollegen gaben für die Niederlande für das Jahr 1988 direkte Kosten des Bronchialkarzinoms in Höhe von 300 Mio. dfl an [14]. Die Hospitalisierungskosten waren auch hier der wichtigste Kostenfaktor.

Den wichtigsten ätiologischen Faktor für die Entstehung des Bronchialkarzinoms stellt der inhalative Nikotinkonsum und die dadurch verursachte chronische Bronchitis dar. Inwieweit und in welcher Richtung die Aufgabe des Rauchens in der Bevölkerung die Kosten im Gesundheitswesen beeinflusst, wird unterschiedlich diskutiert [7]. Barendregt u. Mitarb. 
bildeten ein Modell, das auf der Bevölkerungs- und Kostenstruktur der Niederlande im Jahre 1988 basierte [18]. Sie kamen zu dem Ergebnis, dass auf lange Sicht die komplette Aufgabe des Rauchens zu einem Nettoanstieg der Gesundheitskosten führen würde, bedingt durch die längere Lebenserwartung der Nichtraucher und die sich in den späteren Lebensphasen der Nichtraucher manifestierenden Erkrankungen. Dieses Resultat variierte jedoch mit den Annahmen zum betrachteten Zeithorizont und dem unterstellten Diskontierungssatz. Mit dem Diskontierungssatz wird der gegenwärtige Wert der in künftigen Jahren entstehenden Kosten berechnet. Je höher der Diskontierungssatz gewählt wird, um so niedriger ist der gegenwärtige Wert dieser Kosten. Wie die Autoren selbst ansprechen, fehlt in dieser Betrachtung eine ökonomische Bewertung der durch die Aufgabe des Rauchens gewonnenen Lebensjahre der Bevölkerung. Barendregt u. Mitarb. untersuchten nur direkte Kosten. Dies verbindet sie mit den meisten anderen Studien zur ökonomischen Analyse der Aufgabe des Rauchens. Das auffälligste Ergebnis unserer Krankheitskostenstudie für Deutschland besteht in dem überragenden Anteil der indirekten Kosten in Höhe von 7,40 Mrd. DM an den jährlichen Gesamtkosten des Bronchialkarzinoms in Höhe von 8,31 Mrd. DM. Der größte Kostenfaktor der indirekten Kosten, der vorzeitige Tod, vereinte mit 4,85 Mrd. DM alleine $58 \%$ der Gesamtkosten auf sich. Vor diesem Hintergrund plädieren wir zumindest im Kontext des Bronchialkarzinoms dafür, dass aus volkswirtschaftlicher Perspektive die indirekten Kosten in gesundheitsökonomische Studien zur Aufgabe des Rauchens eingeschlossen werden sollten. Denn sie stellen beim Bronchialkarzinom in charakteristischer Weise den überwiegenden Anteil der Gesamtkosten. Es ist nicht auszuschließen, dass der Einbezug der indirekten Kosten in die einschlägigen ökonomischen Analysen zu einer substantiell anderen Gesamtbeurteilung der Aufgabe des Rauchens führt.

\section{Literatur}

${ }^{1}$ Bundesministerium für Gesundheit (Hrsg). Daten des Gesundheitswesens. Schriftenreihe des Bundesministeriums für $\mathrm{Ge}-$ sundheit. Band 122. Baden-Baden, 1999

${ }^{2}$ Murray CJL, Lopez AD. Alternative projections of mortality and disability by cause 1990 - 2020: Global burden of disease. Lancet 1997; 349: $1498-1504$

${ }^{3}$ Fraumeni JF, Blot WJ. Lung and pleura. In: Schottenfeld D, Fraumeni JF (eds). Cancer epidemiology and prevention. Philadelphia PA: Saunders, 1982: 564-582

${ }^{4}$ Hasse J. Surgical treatment of bronchial carcinoma. Screening methods, early and late results. Berlin, Heidelberg, New York, Tokyo, 1986

${ }^{5}$ Ihde DC. Chemotherapy of lung cancer. N Engl J Med 1992; 327: $1434-1441$

${ }^{6}$ Lange P, Vestbo J. Lung cancer. Eur Respir Mon 2000; 15: 92 104

${ }^{7}$ Goodwin PJ, Shepherd FA. Economic issues in lung cancer: A review. J Clin Oncol 1998; 16: 3900-3912

${ }^{8}$ Copley-Merriman C, Corral J, King K, Whiteside R, Voi M, Dorr A, Mc Donald RC. Economic value of gemcitabine compared to cisplatin and etoposide in non-small cell lung cancer. Lung Cancer 1996; 14: 45-61

${ }^{9}$ Evans WK, Will BP, Berthelot JM, Earle CC. Cost of combined modality interventions for stage III non-small-cell lung cancer. J Clin Oncol 1997; 15: $3038-3048$
${ }^{10}$ Sacristan JA, Kennedy-Martin T, Rossel R, Cardenal F, Anton A, Lomas M, Alberola V, Massuti B, Carrato A, Minshall M. Economic evaluation in a randomized Phase III clinical trial comparing gemcitabine/cisplatin and etoposide/cisplatine in non-small-cell lung cancer. Lung Cancer 2000; 28: 97-107

${ }^{11}$ Younes RN, Gross JL, Deheinzelin D. Follow-up in lung cancer. How often and for what purpose? Chest 1999; 115: 1494-1499

${ }^{12}$ Evans WK, Will BP, Berthelot JM, Wolfson MC. The economics of lung cancer management in Canada. Lung cancer 1996; 14: 19 29

${ }^{13}$ Desch CE, Hillner BE, Smith TJ. Economic considerations in the care of lung cancer patients. Curr Opin Oncol 1996; 8: 126-132

${ }^{14}$ Koopmanschap MA, van Roijen L, Bonneux L, Barendregt JJ. Current and future costs of cancer. Eur J Cancer 1994; 30A (1): 60-65

${ }^{15}$ Rosenthal MA, Webster PJ, Gebski VJ, Stuart-Harris RC, Langlands AO, Boyages J. The cost of treating small-cell lung cancer. Med J Aust 1992; 156: 605-610

${ }^{16}$ Vergnenegre A, Chouaïd C, Lafuma A. L'évaluation médicoéconomique des cancers bronchiques. Approche théorique et applications pratiques. Rev Mal Respir 1998; 15: 469-478

${ }^{17}$ Wolstenholme JL, Whynes DK. The hospital costs of treating lung cancer in the United Kingdom. Br J Cancer 1999; 80: 215-218

18 Barendregt JJ, Bonneux L, van der Maas PJ. The health care costs of smoking. N Engl J Med 1997; 337: 1052 - 1057

${ }^{19}$ Ray GT, Collin F, Lieu T, Fireman B, Colby CJ, Quesenberry CP, van den Eeden SK, Selby JV. The cost of health conditions in a health maintenance organization. Medical Care Research and Review 2000; 57: 92 - 109

${ }^{20}$ Weißbuch Lunge. Defizite, Zukunftsperspektiven, Forschungsansätze; die Lunge und ihre Erkrankungen: Zur Lage der Pneumologie in Deutschland. Konietzko N und Fabel H (Hrsg), erarbeitet von Baur X. Stuttgart, New York, 1996

${ }^{21}$ Weißbuch Lunge. Defizite, Zukunftsperspektiven, Forschungsansätze; die Lunge und ihre Erkrankungen: Zur Lage und Zukunft der Pneumologie in Deutschland. Konietzko N und Fabel H (Hrsg). Stuttgart, New York, 2000

22 Ruff LH, Volmer T, Nowak D, Meyer A. The economic impact of smoking in Germany. Eur Respir J 2000; 16 (3): 385 - 390

${ }^{23}$ Drummond MF. Cost-of-Illness Studies: A major headache? PharmacoEconomics 1992; 2 (1): 1-4

${ }^{24}$ AOK Krankheitsstatistik 1996. AOK-Bundesverband, November 1998

${ }^{25}$ Statistisches Jahrbuch 1998. Statistisches Bundesamt,

${ }^{26}$ VDRSY. Version 1.0. CD-ROM. Der Statistik-Tabellen-Viewer des VDR. Verband Deutscher Rentenversicherungsträger (Hrsg), 1999

${ }^{27}$ Verschreibungsindex für Pharmazeutika. Frankfurt/Main: IMS Health, Auskunft auf Anfrage, Februar 2000

${ }^{28}$ VDR Statistik Rentenzugang 1996. Verband Deutscher Rentenversicherungsträger (Hrsg), Mai 1997

${ }^{29}$ Todesursachen in Deutschland 1996. Wiesbaden: Statistisches Bundesamt. Gesundheitswesen. Fachserie 12, Reihe 4, 1997

\section{Dr. med. Dr. rer. pol. D. Weißflog}

Abteilung für Pneumologie, Medizinische Klinik

Albert-Ludwigs-Universität Freiburg

Hugstetter Str. 55

79106 Freiburg

E-mail: weissflog@g1.ukl.uni-freiburg.de 